International Journal of Distributed and Parallel Systems (IJDPS) Vol.3, No.6, November 2012

\title{
GSM BASED EMBEDDED SYSTEM FOR REMOTE LABORATORY SAFETY MONITORING AND ALERTING
}

\author{
V.Ramya ${ }^{1}$, B. Palaniappan ${ }^{2}$, V.Sumathi ${ }^{3}$ \\ ${ }^{1}$ Assistant Professor, Department of CSE, Annamalai University, Chidambaram, India. \\ ${ }^{1}$ Email:ramyshri@yahoo.com \\ ${ }^{2}$ Dean, FEAT, Head, Department of CSE, Annamalai University, Chidambaram, India. \\ ${ }^{2}$ Email : bpau2002@yahoo.co.in \\ ${ }^{3}$ P.G Scholar, Department of CSE, Annamalai University, Chidambaram, India. \\ ${ }^{3}$ sumathiharini@yahoo.com
}

\begin{abstract}
This paper aims to modify an existing safety and security model for the environment of educational institutions and in home. The aim of this project is to design an embedded system for remote monitoring of the laboratory environment. Nowadays remote monitoring the laboratory and its building is necessary for safety and security purpose, which also help us to know the environmental status of the laboratory. The environmental parameters inside the laboratory, such as presence of alcohol, gas and fire can be detected using respective sensors and the sensed data are then transferred to the microcontroller. The microcontroller takes the control action of activating an alarm whenever the presence of these parameters is found. In turn, the Voice alarm and alert message as SMS through GSM are also sent to the remote area. The advantage of this automated detection and alarm system is that, it offers faster response time and accurate detection during an emergency. Our experimental results show that, the system provides safe and secure remote monitoring of the environment in laboratories and it has high reliability and easy to implement a system like this wherever needed.
\end{abstract}

Keywords - Embedded System, PC16F877, Remote Laboratory, Sensor, Signal conditioning.

\section{INTRODUCTION}

Since the beginning of modern science, alcohol, fire and its co component smoke possess a greater threat to the laboratory and even homes. Many manual technologies are used to counteract and prevent those manmade disasters. The traditional manual data monitoring and management which has low efficiency is still used in many industries as well as homes. With wide ranges of embedded technology and constant improvement of the requirement of embedded applications for laboratory monitoring and management, manual based data monitoring process can no longer meet the requirement of the majority intelligent devices. Traditional data monitoring and management occupies many system resources. Especially in embedded system, hardware and software are limited. The traditional databases cannot be applied on these platforms. This paper focuses the design of the laboratory remote monitoring, which achieves the real time monitoring and gives the automatic voice alarm and alert message to the remote area or host area by using wireless network technology. This work is designed using PIC16F877 microcontroller and focuses on sensing the existence of the critical parameters and issuing the alarm if those parameters were sensed. 


\subsection{Related Work:}

Jiang Linyiang et.al [1] proposed a system which aims at complete monitoring of a variety of real-time data and the states of a laboratory, judging the environmental index automatically, and detecting intrusions from outside which accompanied with sound and light alarm. This system achieves the intelligent management of laboratory. The laboratory monitoring system can communicate with PC, either by wired or wireless means and realize telemetry, meeting the needs of unmanned laboratory.

Christian Trödhandl et.al [2] proposed a system in which the data at the target system was gathered with a time triggered sensor network, which transmits the measured values to the local target server. The sensor network is connected to the target server which communicates with the visualization and programming tools via the web server. The visualization client provides the live display (parameter) of the observed system. The target server act as a gateway between the target system and the monitoring clients, which provides security and authentication features for connecting the monitoring clients. One target server is able to serve for multiple target systems. This approach makes the system easily adaptable to different embedded target systems.

Liu Yang Linyinget.al [3] proposed a system, which provides the software and hardware design solution of an embedded web-based remote laboratory environment monitoring system. This system builds an embedded web server to publish the sensor network data and video images to achieve remote monitoring, which is based on B / S architecture. Managers can control the equipments in the laboratory through a web browser which is a crossplatform. The embedded database manages the data collected by sensor networks, realizing the local management of environmental data. The results show that the designed system implements a safe and convenient remote monitoring and local management of laboratory environment. The system has high availability, reliability and popularization.

Gupta et.al[4] has proposed a system, where tiny wireless sensors embedded in a large number of applications such as, Internet-capable devices-smart phones, cameras, cars, toys, medical instruments, home appliances and energy meters which generates an enormous volume of small bits of data. The real value of this data lies in its analysis, which leads to significant insights and actions that can enhance the health of our planet and its populations. The author has developed a web-based infrastructure made of Sensor Network for storing, sharing, searching, visualizing and analyzing data from heterogeneous devices. This system also facilitates easy interaction amongst devices and end users through an open, REST-based API.

\section{HARDWARE SYSTEM DESIGN}

\subsection{System Design At Laboratory Side}

In the laboratory, the sensor network checks the presence of the parameters such as alcohol, gas and fire. The presence of the alcohol is sensed by the MQ-3 sensor which is also referred as Figaro sensor; the sensed signal is conditioned by the signal conditioning unit and sent to the micro controller. Similarly the fire is sensed through fire sensor and sent to micro controller. The sensed parameters were displayed real time every second in the LCD. In the same way the LPG (Liquefied petroleum gas) is sensed by using MQ-6 sensor and the same process takes place. If any one of the parameter is present, then an alarm is generated immediately. This system may be implemented in the Laboratory to avoid endangering human lives. The block diagram of the proposed system at the laboratory is shown in the Figure 1. 


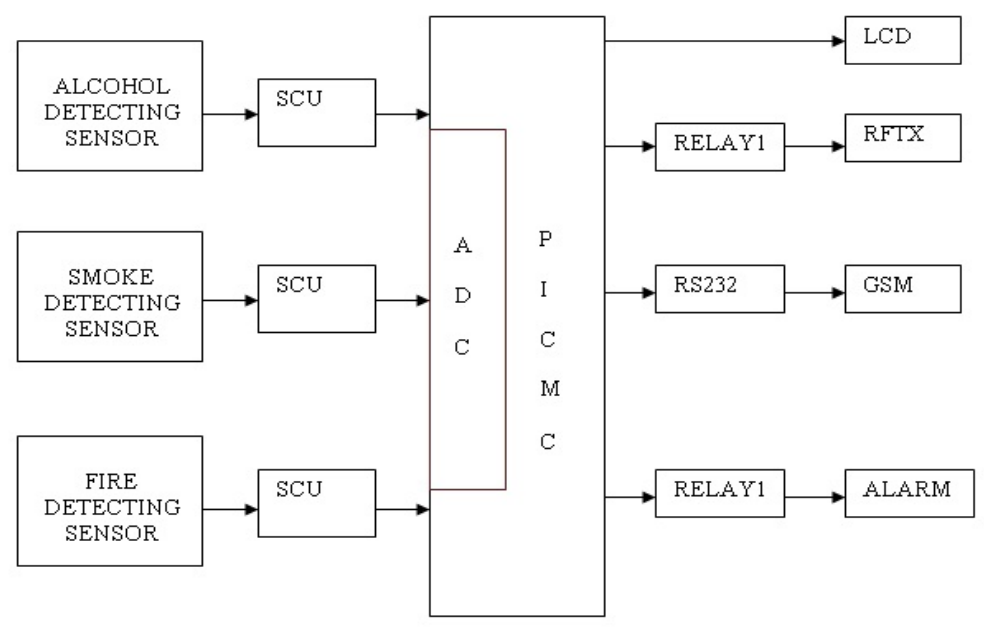

Figure 1. Block diagram of the proposed system at laboratory side system

\subsection{System Design at Remote Side}

In the remote side, the sensed parameters are displayed each and every second in the LCD and a voice alarm is sounded corresponding to the detected parameters. Here the information about the sensed value and the critical situation is received by the RF receiver from the RF transmitter at the laboratory side. The voice alarm is produced with the help of APR 9600 which stores the voices in the specified location and is retrieved back as per the command from the microcontroller. The block diagram of the remote side system is shown in Figure 2.

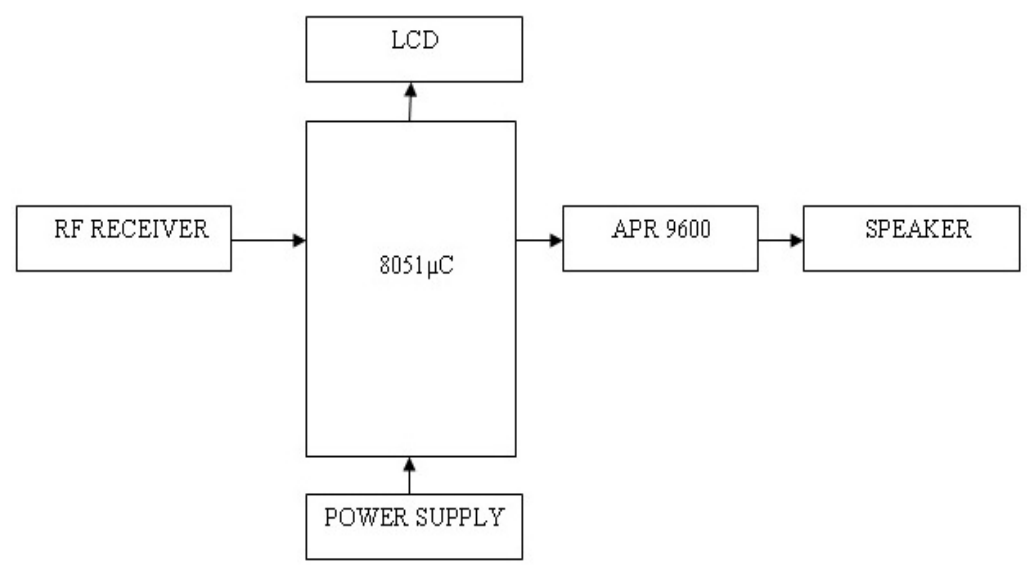

Figure 2. Block diagram of the remote side system

\section{MICROCONTROLLER}

In an embedded system, the Microcontroller is the heart of the system. Hence it must be selected correctly according to the application of the system. There are many families of microcontrollers available such as ATMEL, ATMEGA, and PIC etc. In this work PIC16F877 and 8051 microcontroller were chosen for doing the proposed application. The following are the criteria through which a microcontroller could be selected for a particular system. 
A. Meeting the computing needs of the task efficiently and cost effectively

- Speed, the amount of ROM and RAM, the number of I/O ports and timers, size, packaging, power consumption

- Easy to upgrade

- Cost per unit

B. Availability of software development tools

- Assemblers, debuggers, C compilers, emulator, simulator, technical support

C. Wide availability and reliable sources of the microcontrollers

\subsection{PIC16F877}

At the laboratory side PIC16F877 is used. PIC16F877 forms the heart of this system. It consists of clock circuit and power on reset circuit. The Clock circuit is built around a crystal oscillator and a ceramic capacitor. The purpose of the crystal oscillator is to stabilize the frequency and the capacitor is to stabilize the amplitude of the clock. This circuit determines the operating speed. Here we use $4 \mathrm{MHz}$ crystal oscillator, so the microcontroller will work at the speed of 1 microSec.

\subsubsection{Chip features}

- High-Performance RISC CPU

- Only 35 single-word instructions to learn

- 10-bit, up to 8-channel Analog-to-Digital Converter (A/D)

- Self-reprogrammable under software control

- Operating speed: $\mathrm{DC}-20 \mathrm{MHz}$ clock input

- Low-power consumption

- Up to $8 \mathrm{~K}$ x 14 words of Flash Program Memory

- Up to $368 \times 8$ bytes of Data Memory (RAM)

- Up to 256 x 8 bytes of EEPROM Data Memory

- 14 interrupts, 3 timers

\subsection{Microcontroller}

The 8051 is a single-chip 8-bit microcontroller which executes ASM51 instruction set. The microcontroller supports serial communication interface, timer, multi-purpose I/O ports, hardware interrupts and debugger interface. Target applications for the 8051 are those in which the microcontroller replaces hard-coded control logic which is being easily re-programmable and also allows modifications to the control algorithm without re-designing the chip. At remote side, 8051 microcontroller is chosen, because there only small amount of functions like receiving the RF signal, displaying the sensed parameter and providing the voice alarm are performed. It does not require more ports and memory.

\section{LABORATORY SIDE COMPONENTS DESCRIPTION}

\subsection{Alcohol Sensing System}

The alcohol sensor MQ-3 is suitable for detecting alcohol concentration on our breath, just like the common breathalyser. It has a high sensitivity and fast response time. Sensor provides an analogue resistive output based on alcohol concentration. The drive circuit is very simple and it needs is one resistor. A simple interface could be a $0-3.3 \mathrm{~V}$ A. The alcohol sensing module is shown in figure 3. Interfacing alcohol sensor with microcontroller is shown in figure 4. 
International Journal of Distributed and Parallel Systems (IJDPS) Vol.3, No.6, November 2012

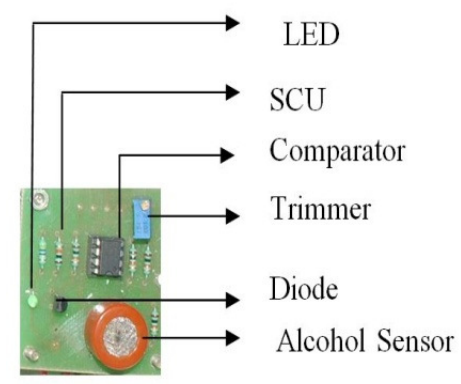

Figure 3. Alcohol sensing system

Table.1: Specifications of the Alcohol Sensing System

\begin{tabular}{|l|l|l|}
\hline \multicolumn{1}{|c|}{ Parameter } & \multicolumn{1}{|c|}{ Value } & \multicolumn{1}{c|}{ Unit } \\
\hline Target Gas & Alcohol & PPM (parts per million) \\
\hline Detection Range & $0.05 \mathrm{mg} / \mathrm{L}-10 \mathrm{mg} / \mathrm{L}$ & Milligram per Litre \\
\hline Output Voltage Range & 0 to 5 & V \\
\hline Working Voltage & 5 & V \\
\hline Current Consumption & $<180$ & $\mathrm{~mA}$ \\
\hline Warm up Time & 10 & Minutes \\
\hline Calibrated Gas & $0.4 \mathrm{mg} / \mathrm{L}$ & Alcohol \\
\hline Response Time & $<10 \mathrm{~s}$ & Seconds \\
\hline Resume Time & $<30 \mathrm{~s}$ & Seconds \\
\hline $\begin{array}{l}\text { Standard Working } \\
\text { Condition }\end{array}$ & Temperature:-10 to $65^{\circ} \mathrm{C}$ & ${ }^{\circ} \mathrm{C}$ \\
\hline Storage Condition & Humidity: $<95 \% \mathrm{RH}$ & ${ }^{\circ} \mathrm{C}$ \\
\hline
\end{tabular}




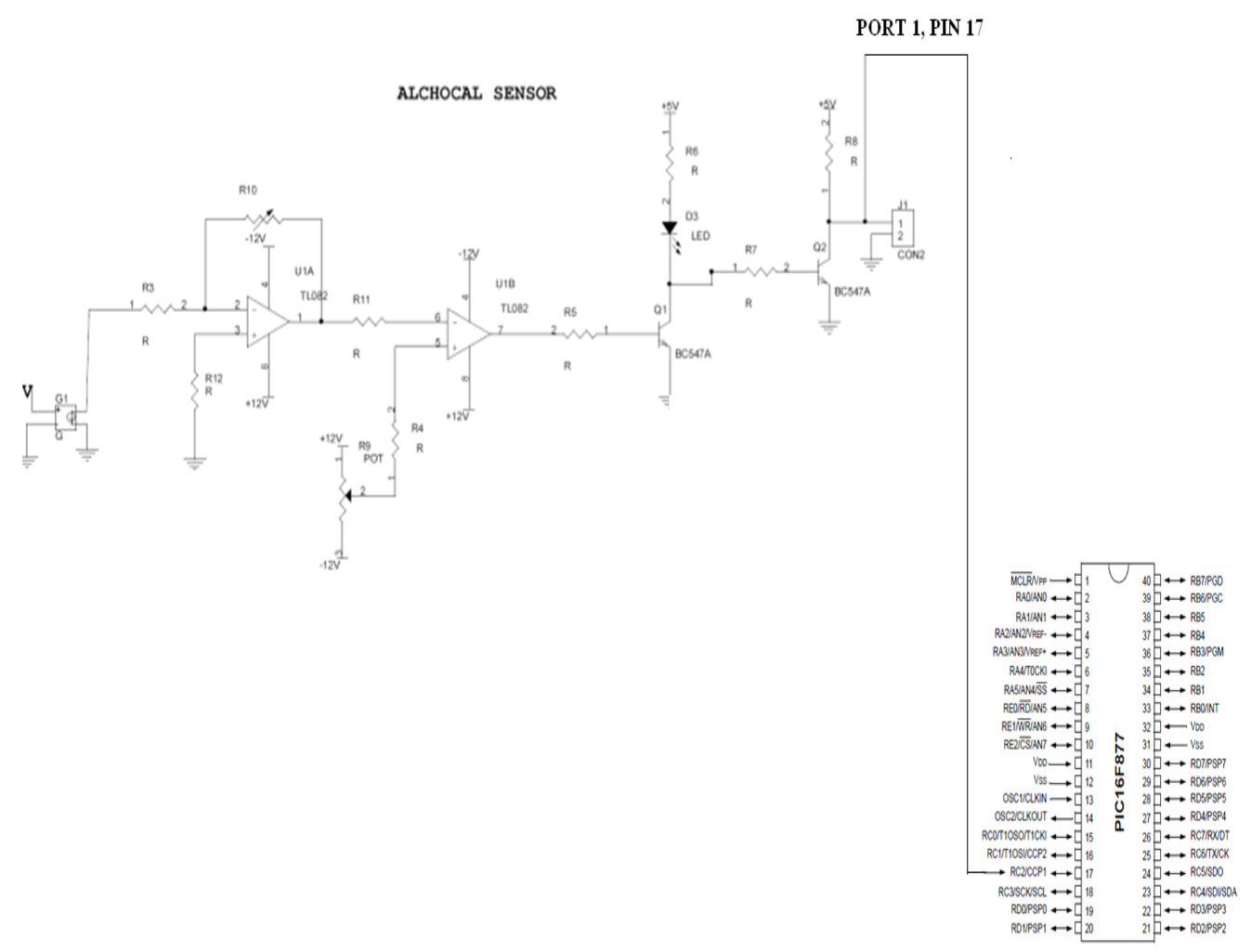

Figure 4. Interfacing alcohol sensor with PIC microcontroller

\subsubsection{Features}

- Low power consumption

- High sensitivity to gaseous air contaminants

- Long life and low cost

- Uses simple electrical circuit

\subsubsection{Applications}

- Air cleaners

- Ventilation control

- Air quality monitors

\subsection{Gas Sensing System}

The sensing element comprised of a metal oxide semiconductor layer which is formed on an alumina substrate of a sensing chip along with an integrated heater in the presence of a detectable gas. The sensor's conductivity increases depending on the air. A simple electrical circuit can convert the change in conductivity to an output signal which corresponds to the gas concentration. The sensor can detect hydrogen at several ppms. Figure 5 shows the Gas detection sensor module. Interfacing gas sensor with microcontroller is shown in figure 6 . 
International Journal of Distributed and Parallel Systems (IJDPS) Vol.3, No.6, November 2012

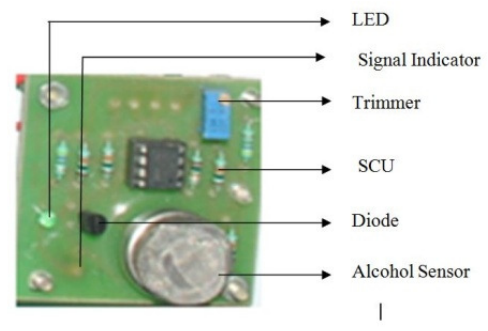

Figure.5 Gas Sensing System

Table.2: Specifications of the Gas Sensing System

\begin{tabular}{|c|c|c|c|c|}
\hline \multicolumn{3}{|l|}{ Model number } & \multicolumn{2}{|l|}{ TGS 2600} \\
\hline \multicolumn{3}{|c|}{ Sensing element type } & \multicolumn{2}{|l|}{ D1 } \\
\hline \multicolumn{3}{|l|}{ Standard package } & \multicolumn{2}{|l|}{ TO-5 metal can } \\
\hline \multicolumn{3}{|l|}{ Target gases } & \multicolumn{2}{|l|}{ Co } \\
\hline \multicolumn{3}{|c|}{ Typical detection range } & \multicolumn{2}{|l|}{$1 \sim 10 \mathrm{ppm}$ of $\mathrm{H}_{2}$} \\
\hline \multirow{3}{*}{$\begin{array}{l}\text { Standard circuit } \\
\text { conditions }\end{array}$} & Heater voltage & $\mathrm{V}_{\mathrm{H}}$ & \multicolumn{2}{|c|}{$5.0 \pm 0.22 \mathrm{~V} \mathrm{DC} / \mathrm{AC}$} \\
\hline & Circuit voltage & $\mathrm{V}_{\mathrm{C}}$ & $5.0 \pm 0.22 \mathrm{~V} \mathrm{DC}$ & Ps $\leq 15 \mathrm{~mW}$ \\
\hline & Load resistance & $\mathrm{R}_{\mathrm{L}}$ & Variable & Ps $\leq 15 \mathrm{~mW}$ \\
\hline \multirow{5}{*}{$\begin{array}{l}\text { Electrical } \\
\text { characteristics } \\
\text { under standard } \\
\text { test } \\
\text { Conditions }\end{array}$} & Heater resistance & $\mathrm{R}_{\mathrm{H}}$ & \multicolumn{2}{|c|}{$83 \Omega$ at room temp. (typical ) } \\
\hline & Heater current & $\mathrm{I}_{\mathrm{H}}$ & \multicolumn{2}{|l|}{$42 \pm 4 \mathrm{~mA}$} \\
\hline & Heater power consumption & $\mathrm{P}_{\mathrm{H}}$ & \multicolumn{2}{|l|}{ 210mW (typical) } \\
\hline & Sensor resistance & $\mathrm{R}_{\mathrm{S}}$ & \multicolumn{2}{|l|}{$10 \sim 90 \mathrm{k} \Omega$ in air } \\
\hline & \multicolumn{2}{|c|}{ Sensitivity (change ratio of Rs.) } & $0.3 \sim 0.6$ & $\begin{array}{l}\text { s. }\left(10 \mathrm{ppm} \text { of } \mathrm{H}_{2} \text { ) }\right. \\
\text { s. (air) }\end{array}$ \\
\hline \multirow{2}{*}{$\begin{array}{ll}\text { Standard test } \\
\text { conditions }\end{array}$} & \multicolumn{2}{|l|}{ Test gas conditions } & \multicolumn{2}{|c|}{$\begin{array}{l}\text { Normal air } \\
\text { At } 20 \pm 2^{0} \mathrm{C}, 65 \pm 5 \% \mathrm{RH}\end{array}$} \\
\hline & \multicolumn{2}{|l|}{ Circuit conditions } & \multicolumn{2}{|c|}{$\begin{array}{l}\mathrm{Vc}=5.0 \pm 0.01 \mathrm{VDC} \\
\mathrm{V}_{\mathrm{H}}=5.0 \pm 0.05 \mathrm{VDC}\end{array}$} \\
\hline
\end{tabular}




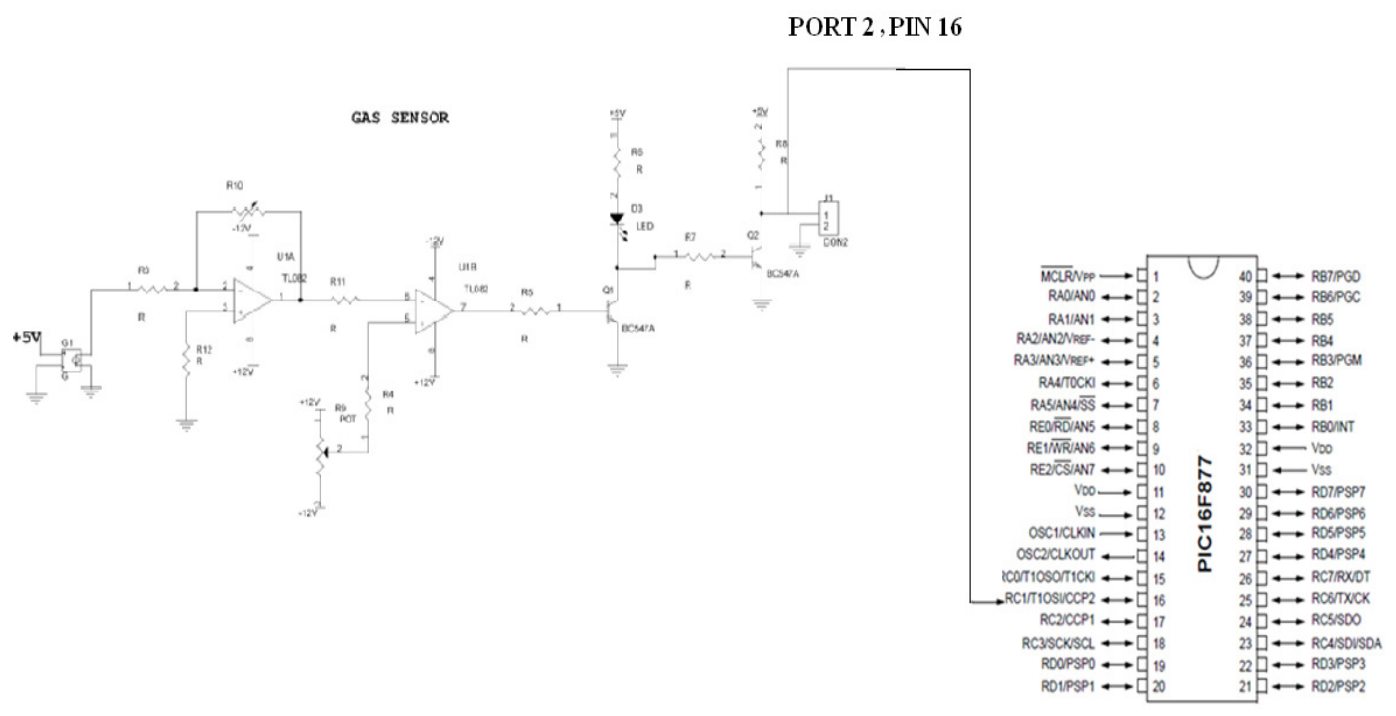

Figure 6. Interfacing gas sensor with PIC Microcontroller

\subsubsection{Features}

- Low power consumption

- High sensitivity to gaseous air contaminants

- Long life and low cost

- Uses simple electrical circuit

\subsection{Fire Sensing System}

Thermocouples, Resistance Temperature Detectors (RTDs), Thermistors, and Semiconductor Sensors are the different varieties of sensors which are readily available. In our work, Thermistor is used. Fire sensing module is shown in figure 7, and interfacing with microcontroller is shown in figure 8 .

\subsubsection{Thermistor}

Thermistor is a temperature sensing device whose resistance changes with temperature. Thermistors, however, are made from semiconductor materials. Thermistors exhibit a highly nonlinear resistance versus temperature curve. In the Thermistor's operating range, a large resistance change can be observed for a very small temperature change. This makes it a highly sensitive device, which is ideal for set-point applications. Fire sensing module is shown in figure 7, and interfacing fire sensor with microcontroller is shown in figure 8. 


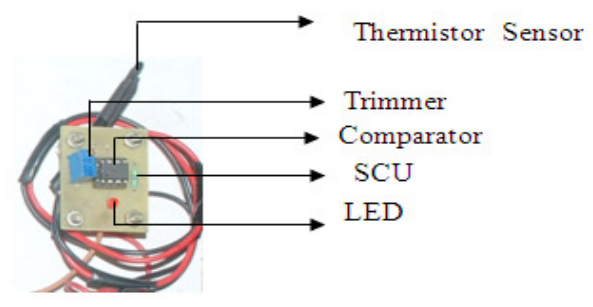

Figure 7. Fire Sensing System

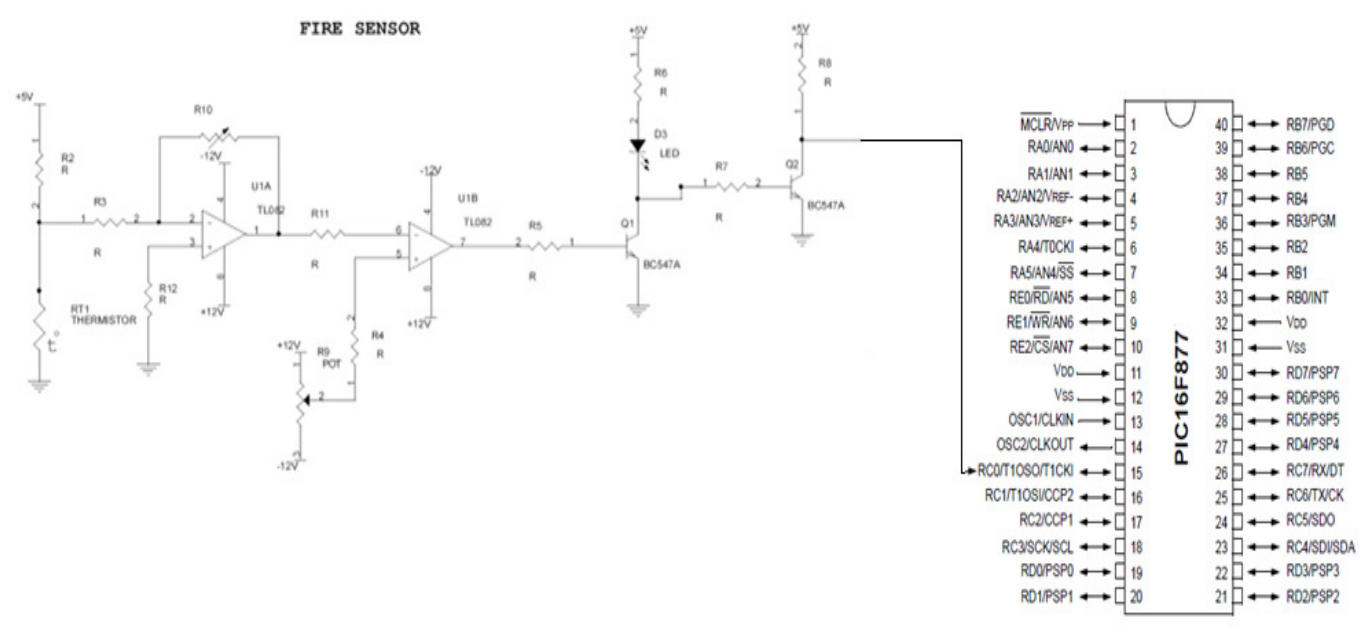

Figure 8. Interfacing fire sensor with PIC microcontroller

Thermistor requires external electrical excitation and significant signal conditioning. Chips exist which can convert a Thermistor value directly to digital data like the chip with I2C serial interface, but currently it is not widely available. Simple circuits can be built to read in the Thermistor voltage, which can be fed to an ADC, and the temperature determined digitally.

The ceramic Thermistor devices can be further subdivided into two categories

1. NTC (Negative Temperature Coefficient Thermistor)

2. PTC (Positive Temperature Coefficient Thermistor)

\subsubsection{NTC (Negative Temperature Coefficient Thermistor)}

The first category consists of components whose resistance decreases as their temperature increases; i.e. Thermistor components with a Negative Temperature Coefficient, known as NTC Thermistors. For temperature sensing applications with wide temperature range of $-80^{\circ} \mathrm{C}$ to $+250^{\circ} \mathrm{C}$ it is ideal to use NTC Thermistors. Our study uses the above said NTC Thermistor.

\subsubsection{PTC (Positive Temperature Coefficient Thermistor)}

The second category consists of components whose resistance increases as their temperature increases which is known as PTC Thermistors. These are the low cost and high 
resistance sensor, typically used for circuit protection applications rather than temperature sensing applications.

\subsection{LCD Display}

LCDs are used as numerical indicators; especially in digital watches where there is a much smaller current needed than LED displays (microamperes compared with milliamperes) which prolongs battery life. Liquid crystals are organic (carbon) compounds, which exhibit both solid and liquid properties. The LCD display used in this project consists of 2 rows. Each row consists of maximum 16 characters. So using this display, a maximum of 32 characters can be displayed. The LCD display is shown in figure 9.

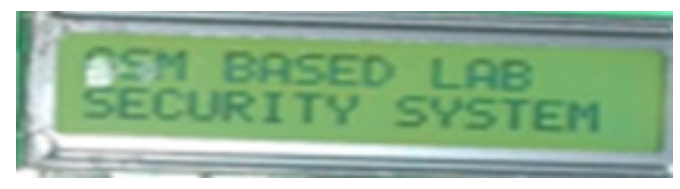

Figure 9. LCD Display

\subsection{RS232}

The GSM modem works on the RS-232 voltage levels. Its 'logic 1' varies from -3 to 15 Volts and 'logic 0 ' from +3 to +15 Volts. A microcontroller works on TTL logic levels and its ' $\operatorname{logic} 1$ ' is +5 Volts and 'logic 0 ' is 0 Volts. Therefore to interface both, we use a MAX 232 driver IC manufactured by Maxim.

\subsection{Buzzer Alarm}

A buzzer is a signaling device, usually electronic, typically used in automobiles, household appliances such as a microwave oven, or game shows. It most commonly consists of a number of switches or sensors connected to a control unit that determines which button was pushed or a preset time has lapsed. A light will be illuminated on the appropriate button or control panel, and raises the warning in the form of a continuous or intermittent beep sound. The alarm module is shown in figure 10. The Interfacing Alarm with PIC Microcontroller is shown in figure 11.

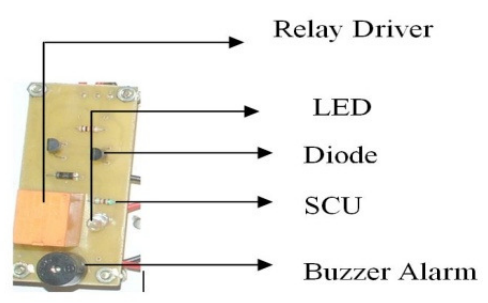

Figure 10. Buzzer Alarm 


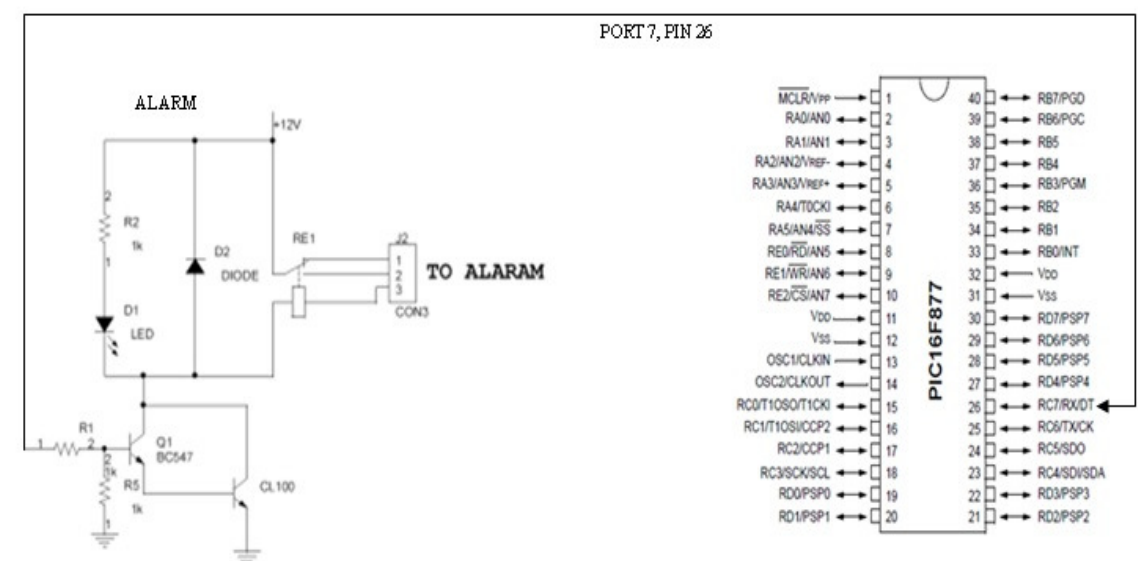

Figure 11. Interfacing Alarm with PIC Microcontroller

\subsection{RF Communication Module}

The RF module operates at the Radio Frequency and its frequency range varies between 30 $\mathrm{kHz}$ to $300 \mathrm{GHz}$. The digital data is represented as variations in the amplitude of carrier wave and this kind of modulation is called as Amplitude Shift Keying (ASK). The RF Transmission is better than IR (infrared) because of many reasons.

- Signals through RF can travel through longer distances making it suitable for long range applications.

- IR mostly operates in line-of-sight mode but RF signals can travel even when there is an obstruction between transmitter and receiver.

- $\quad \mathrm{RF}$ transmission is more strong and reliable than IR transmission.

- RF communication uses a specific frequency unlike IR signals which are affected by other IR emitting sources.

This RF module comprises of a RF Transmitter and a RF Receiver. The transmitter/receiver $(\mathrm{Tx} / \mathrm{Rx})$ pair operates at a frequency of $434 \mathrm{MHz}$ and $\mathrm{RF}$ transmitter receives serial data and transmits it wirelessly through RF via an antenna connected at pin4. The transmission occurs at the rate of $1 \mathrm{Kbps}-10 \mathrm{Kbps}$. The transmitted data is received by the RF receiver operating at the same frequency as that of the transmitter. The RF module is often used along with a pair of encoder/decoder. The encoder is used for encoding parallel data for transmission feed, while reception is decoded by a decoder. HT12E-HT12D, HT640-HT648, etc. are some commonly used encoder/decoder pair ICs.

\subsection{GSM Modem}

A GSM (Global System for Mobile Communication, originally from Group Special Mobile) modem is a wireless modem that works with a GSM wireless network. A wireless modem behaves like a dial-up modem, except the main difference between them is that a dialup modem sends and receives data through a fixed telephone line, while a wireless modem 
sends and receives data through radio waves. Like a GSM mobile phone, a GSM modem requires a SIM card from a wireless carrier in order to operate. A GSM modem can be an external unit or a PCMCIA card (also called PC Card). An external GSM modem is connected to a PC through either a serial cable or a USB cable or Bluetooth or Infrared. GSM is the most popular standard for mobile phones in the world. It is used by over 3 billion people across more than 212 countries and territories. Its ubiquity makes international roaming very common between mobile phone operators, enabling subscribers to use their phones in many parts of the world. GSM digitalize both signals and speech channels and thus is considered as a second generation mobile phone system. This has also meant that data communication was easy to build into the system. The GSM module is shown in figure 12 .

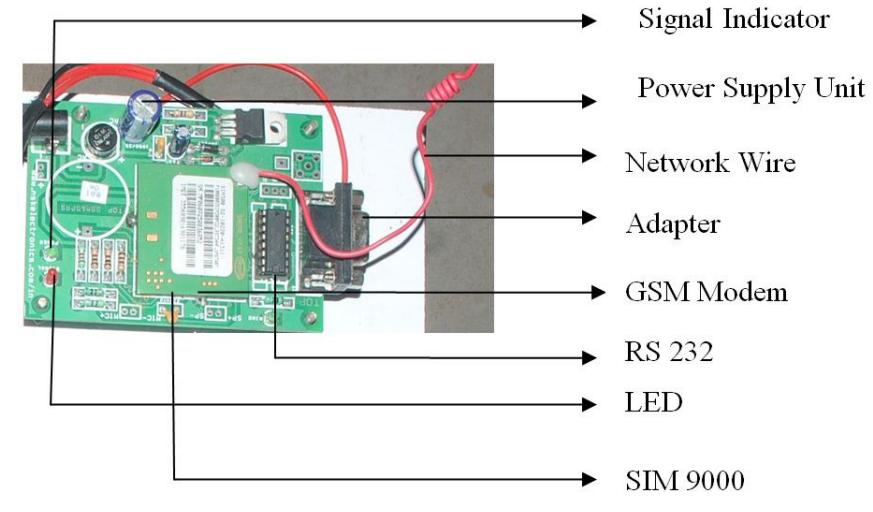

Figure 12. GSM Modem

\section{AT Commands}

The Microcontroller uses the AT commands to control the GSM modem. GSM modems and normal Hayes modems support a common set of AT commands. GSM modem supports an extended set of AT commands which are defined in the GSM standards. With these we can:

- Send SMS messages. Monitor the signal strength.

- The battery charge level and its charging status also be monitored.

- Read, write and search phone book entries.

\section{General Syntax}

To control a modem AT commands are used. AT is the abbreviation of ATtention. Every command line starts with "AT" or "at". That's why modem commands are called AT commands. The starting of a command line is recognized by the modem with help of the prefix "AT" and is not a part of the AT command name. For example, 'D' is the actual AT command name in 'ATD' and '+CMGS' is the actual AT command name in 'AT+CMGS'. Many of the commands which are used to control wired dial-up modems, such as ATD (Dial), ATA (Answer), ATH (Hook control) and ATO (Return to online data state), are also supported by GSM/GPRS modems and mobile phones. Interfacing GSM Modem with PIC Microcontroller is shown in the figure 13 . 

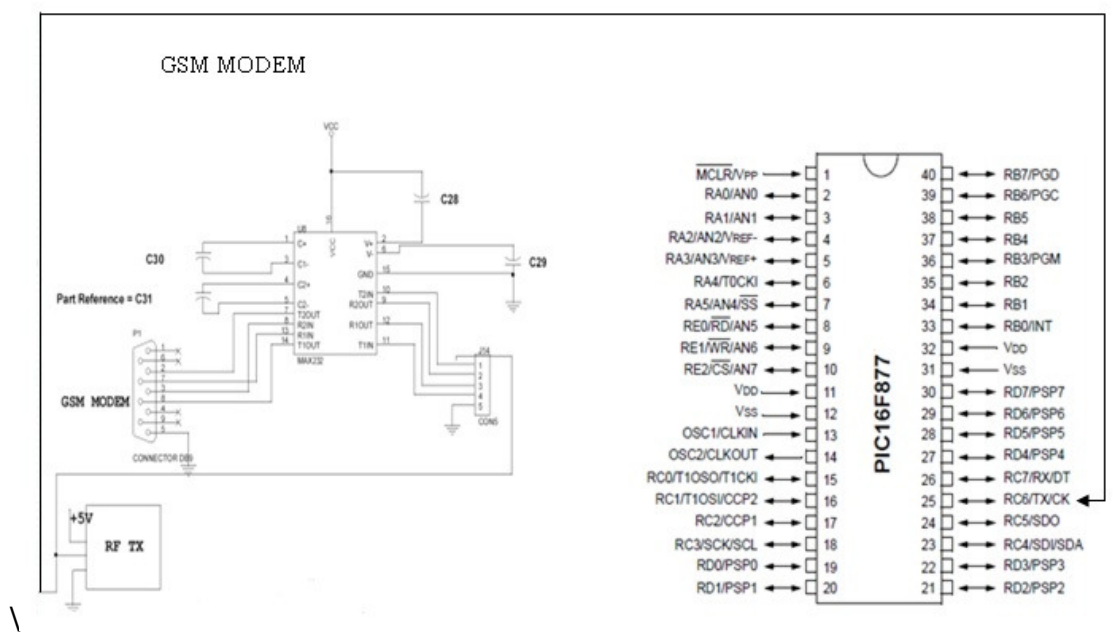

Figure 13. Interfacing GSM Modem with PIC Microcontroller

\section{Sending Messages}

To send SMS messages, first a valid SIM card from a wireless carrier is placed into a mobile phone or GSM/GPRS modem, which is then connected to a computer. A mobile phone or GSM/GPRS modem can be to a computer by several ways. For example, they can be connected through a serial cable, a USB cable, a Bluetooth link or an infrared link.

The instructions used for controlling the mobile phone or GSM/GPRS modem are the AT commands (AT commands are also used to control dial-up modems for wired telephone system). In addition to the common set of standard AT commands, mobile phones and GSM/GPRS modems support an extended set of AT commands. The extended AT commands is to control the sending and receiving of SMS messages. The following table lists the AT commands that are related to the writing and sending of SMS messages:

Table 3. AT commands

\begin{tabular}{|c|c|}
\hline AT command & Meaning \\
\hline $\mathrm{AT}+\mathrm{CMGS}$ & Send message \\
\hline $\mathrm{AT}+\mathrm{CMSS}$ & $\begin{array}{c}\text { Send message from } \\
\text { storage }\end{array}$ \\
\hline $\mathrm{AT}+\mathrm{CMGW}$ & $\begin{array}{c}\text { Write message to } \\
\text { memory }\end{array}$ \\
\hline $\mathrm{AT}+\mathrm{CMGD}$ & Delete message \\
\hline $\mathrm{AT}+\mathrm{CMGC}$ & Send command \\
\hline $\mathrm{AT}+\mathrm{CMMS}$ & More messages to send \\
\hline
\end{tabular}


International Journal of Distributed and Parallel Systems (IJDPS) Vol.3, No.6, November 2012

\section{REMOTE SIDE COMPONENTS DESCRIPTION}

\subsection{Comparator LM358}

The LM2904, LM358/LM358A, LM258/LM258A consist of two independent, high gain, internal frequency Compensated operational amplifiers which were designed specifically to operate from a single power supply over a wide range of voltage. Operation from split power supplies is also possible and the low power supply current drain is independent of the magnitude of the power supply voltage. Application areas include transducer amplifier, DC gain blocks and all the conventional OP-AMP circuits which now can be easily implemented in single power supply systems.

\subsection{Voice Alarm}

\subsubsection{APR9600}

The APR9600 is a 28 pin device used to record and playback maximum of 8 messages. The device offers true single-chip voice recording, non-volatile storage, and playback capability for 40 to 60 seconds. It also supports both random and sequential access of multiple messages. Sample rates are user-selectable and allow the designers to customize their design for unique quality and storage time needs. Its storage technology is vast and it can store up to 256 voltage levels. Hence the APR9600 is enables to reproduce the voice signals in their natural form, eliminating the need for encoding and compression, which often introduce distortion. Figure 14 shows the APR9600. Figure 15 shows the pin diagram of APR9600.

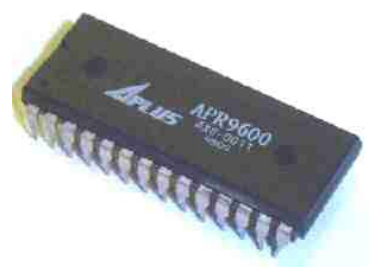

Figure 14. APR 9600

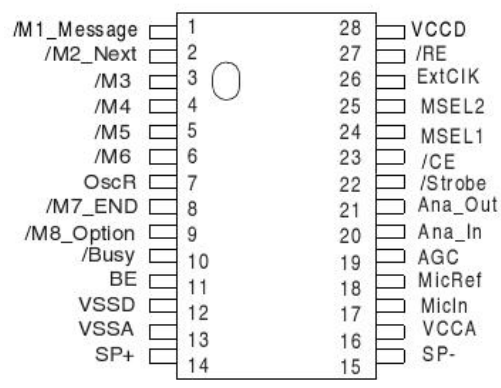

Figure 15. Pin Diagram of APR9600 


\section{Record Mode (Level-Activated)}

In record mode a single voice message of up to 20 seconds can be recorded. The LED pin will glow during the actual recording process to provide a visual indication. As long as the Rec L pin stays low (level-activated) then the chip will be in record mode. The recording will terminate automatically whenever the message lasts longer than 20 seconds after the last available memory cell is written. If the message is shorter than 20 seconds, the recording operation will stop and the/Rec L pin goes high. The speaker driver is automatically restarted during the recording operation. Messages of up to 30 seconds can be recorded by using different resistor values.

\section{Playback Mode (Edge-Activated)}

The chip is in playback mode after the PlayE pin pulses low (edge-activated). Playback will stop immediately whenever the PlayE pin pulses low at second time. If the newly recorded message is shorter than the previously recorded message, then the remaining portion of the previous message will not be played after the new message was played back. The input preamplifier, AGC, and main amplifier circuits are disabled during playback. At Standby Mode $(\mathrm{CE}=" 0 ")$, the chip will automatically return to the standby state, whenever the recording or playback operation is completed. At Power down Mode (CE = "1"), the chip is always in standby state. No recording or playback is allowed. Current consumption is typically less than 1 .

Voice signal from the microphone as shown in figure.15 from the remote side of the prototype is fed into the chip through a differential amplifier. It is further amplified by connecting analog output (pin 21) to analog input (pin 20) via an external blocking capacitor $\mathrm{C} 1$. A bias signal is applied to the microphone and to save power during play back, the ground return of this bias network can be connected to the normally open side of the record switch. Both microphone input (Mic.In) and Microphone reference (Mic. Ref) (pins 18and 19) must be coupled. Analogue storage technique is implemented using flash non-volatile memory in which each cell is capable of storing up to 256 voltage levels. This technology enables the APR9600 to reproduce voice signals in their natural form with play back capability for 32 to 60 seconds. Microphone amplifier, automatic gain control (AGC) circuits, internal anti-aliasing filter, integrated output amplifier and message managements are some of the features of the APR9600 chip.

\section{SOFTWARE SYSTEM DESIGN}

\subsection{Keil Compiler}

Keil provides several development tools like

- IDE (Integrated Development Environment)

- Project Manager

- Simulator

- Debugger

- C Cross Compiler, Cross Assembler, Locator/Linker

Keil provides following tools for 8051 development [4].

A project development cycle: - these are the steps to develop 8051 project using keil

1. Create source files in $\mathrm{C}$ or assembly 
2. Compile or assemble source files

3. Correct errors in source files

4. Link object files from compiler and assembler

5. Test linked application

\subsection{Embedded C}

Embedded $\mathrm{C}$ is a set of language extensions for the $\mathrm{c}$ programming language by the $\mathrm{C}$ standards committee. It introduces a number of features not available in normal $\mathrm{C}$ and basic $\mathrm{I} / \mathrm{O}$ hardware addressing [8]. It is having the declaration of microcontroller registers and special function as header files, we can include these files to make easy implementation. Embedded $\mathrm{C}$ has same flow and programming methodology as C. It has unlimited number of source files, mixed $\mathrm{C}$ and assembler programming. Key characteristics of an embedded system, when compared to PCs, are as follows:

- Embedded devices have resource constraints (limited ROM, limited RAM, and limited stack space, less processing power)

- Embedded systems are more tied to the hardware and typically use smaller and less power consuming components.

Use of $\mathrm{C}$ in embedded systems is driven by the following advantages

- It is simple to learn, understand, program and debug.

- C Compilers are available for almost all embedded devices, and there is a large pool of experienced $\mathrm{C}$ programmers.

- As C combines functionality of assembly language and features high level languages, $\mathrm{C}$ is treated as a 'middle-level computer language' or 'high level assembly language'

- It is fairly efficient

- It supports accessing to the $\mathrm{I} / \mathrm{O}$ and provides easy of management of large embedded projects.

\section{IMPLEMENTATION}

\subsection{Prototype of the Laboratory Side System}

The power supply unit converts AC to DC using bridge rectifier. Alcohol sensor senses the presence of alcohol and sends signal to the microcontroller. Similarly, gas and temperature were sensed and signals sent to the microcontroller. If any one of the parameter is present, then the LCD displays the presence of the parameter and the alarm makes a beep sound. At the laboratory side, the RF transmitter transmits the message to the authorized person/persons at remote side using GSM modem. The Flow chart of the Laboratory side and Remote side is shown in figure 16 and 17. The Prototype of the laboratory side system is shown in figure 18. 
International Journal of Distributed and Parallel Systems (IJDPS) Vol.3, No.6, November 2012

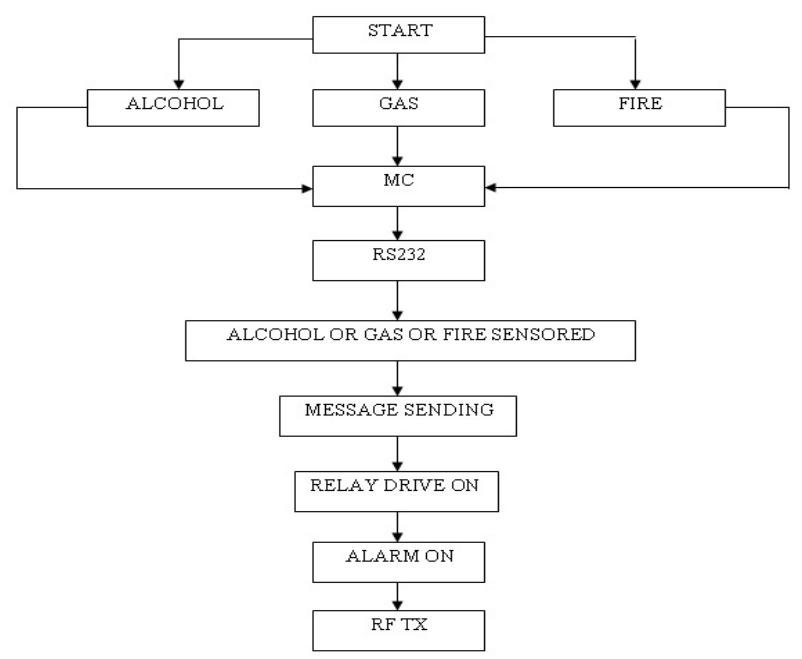

Figure 16. Flow chart of the Laboratory Side

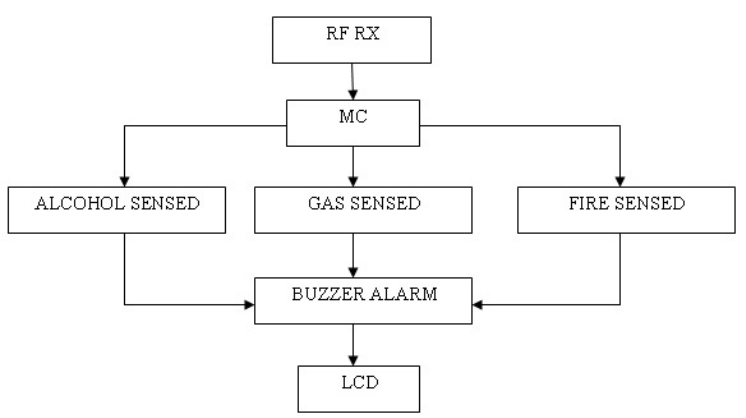

Figure 17. Flow chart of the Remote Side

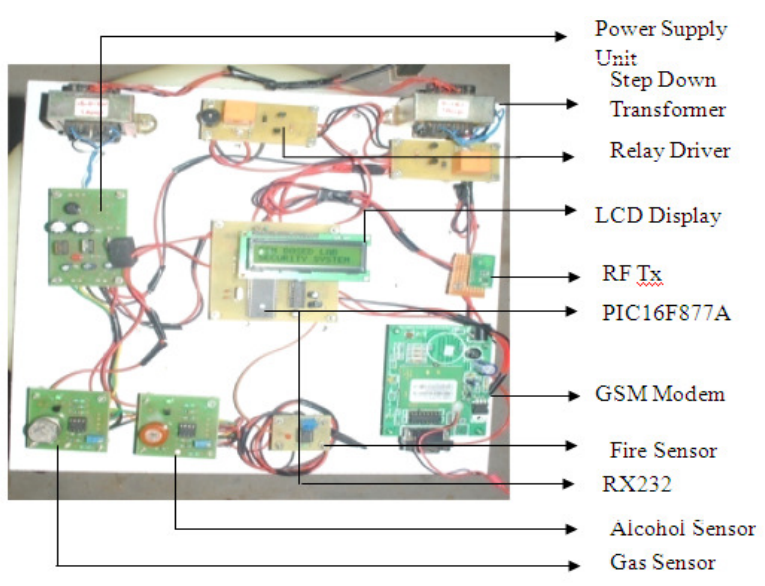

Figure 18. Prototype of the laboratory side system 


\subsection{Prototype of the Remote Side System}

In remote side, RF receiver is used to receive the information. If any one of the parameter received in remote side of the RF receiver, it signals the 8051 microcontroller. The Microcontroller automatically provokes buzzer alarm, when it receives the parameters like Gas $=\mathrm{A}$, Alcohol $=\mathrm{B}$ and Fire $=\mathrm{C}$ and the $\mathrm{LCD}$ displays the received information in remote side. The Prototype of the remote side system is shown in the figure 19.

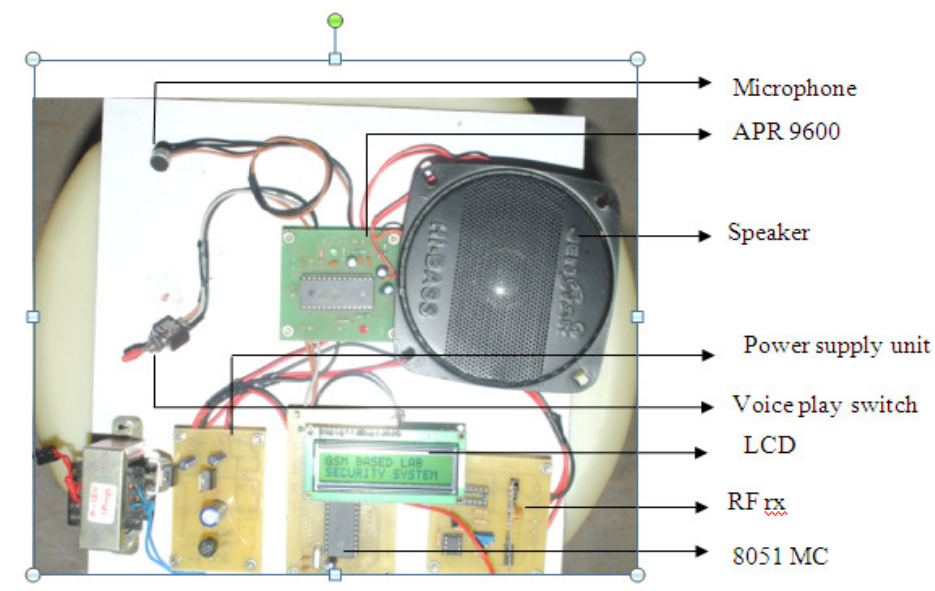

Figure 19. Prototype of the remote side system

\section{CONCLUSION}

Each and every lab stores years of many peoples' hard work. A fire or a gas leak destroys not only valuable resources, but also the hard work of many such persons. One laboratory has been installed our system. Our system of implementing sensors and the other modules is a successful venture. It detects the biohazards like Alcohol, fire or gas leaks and once detected, the sensors relays the information and the particular hazard is displayed in the laboratory using LCD. The responsible persons are alerted through the RF and GSM technology both in site (lab) and off site (remote). Early warning about these hazards at the neck of time can save precious resources and valuable lives. The same could be implemented in real time such that, the hardware configuration of the sensors could be enhanced in a way that it fits exactly with the Laboratory remote monitoring to be implemented. In future the distance between the laboratory and the remote monitoring can be increased by using Zigbee technology.

\section{REFERENCES}

1. Jiang Linyiang,Li Hailong and Guo Zhenhua, IEEE "Embedded Laboratory Environment Monitor System”, IEEE, WASE International Conference on Information Engineering,pp.197201,2009 .

2. Christian Tr"odhandl, Markus Proske, and Wilfried Elmenreich, IEEE "Remote Target Monitoring in Embedded Systems Lab Courses using a Sensor Network”,pp.5433-5438,2006,

3. Gupta, V. Poursohi, A. Udupi,P. Sun Microsyst. Labs and Menlo Park, CA, USA,IEEE "Sensor. Network: An open data exchange for the web of things".pp.753-755,2010. 
International Journal of Distributed and Parallel Systems (IJDPS) Vol.3, No.6, November 2012

4. Wang ping, wany Zheng, "IEEE, design and Implementation of open computer lab monitoring and management system" IEEE, computer and modernization,11,pp.125-128. 2007.

5. Tan Jiyuan, Wu Chengdong, "Research of Abnormal Target Detection Algorithm in infelignt surveillance system”, Electrical and Mechanical Engineering,26(3),pp.12-15, 2009.

6. Jing li, yong xu, "Remote Monitoring systems Based on Embedded Database", third International conference on Genetic and evolutionary computing",pp.381-384,

7. Wang Guowei, song Techeng, Chen Zhengshi."'Surveillance servers based on embedded web Technology", 31 (22),pp.202-204.

8. Kun Yue, Linying Jiang, Liu Yang, Heming Pang, "Research of Embedded database SQLite Application in Intelligent Remote Monitoring System”,2010.

9. Hee $\mathrm{s}$, hee $\mathrm{KC}$, "Integration of mobile vehicles for automated material handling using profibus and IEE 802-11 networks", IEEE, Transactions on Industrial Electronics, 2002, 693-701. In the year of 2007 ,

10. Jiang hinying, Li dAncheng, wang ping, "Design and Implementation SMS based HIRELESS Remote monitoring centre”. mini-micro systems, 2006,

11. Christian trodhandl, markus proske, and wilfried elmenreich vienna university“Remote target monitoring in embedded systems lab courses using a sensor network" 2006.

12. Liu Yang, Heming Pang, Linying Jiang ,Kun Yue ,'The Research of Embedded Linux and SQLite Database Application in the Intelligent Monitoring System". Northeastern University,2010.

13. Cristina Anita Bejan, Mihai Iacob and Gheorghe-Daniel Andreescu," SCADA Automation System Laboratory, Elements and Applications", "Politehnica" University of Timisoara, Dept. of Automation and Applied Informatics, Timisoara, Romania, 181-186,2009.

14. Guozheng Li and Nanlin Tan, IEEE "Design and Implementation of Remote Monitoring and Control System for Freight Train Load Testing”, 2010.

15. Michael J. McGrath, Michael J. McGrath and John Delaney, "An Extensible Framework for the Management of Remote Sensor Data”, Health Research and Innovation, IEEE,1-4. 2011.

16. Wang ping, wanyZheng, 'Design and Implementationof open computer lab monitoring and management system', computer and modernization, IEEE ,11,pp.125-128, 2007. 